\title{
Aneurisma aórtico abdominal en pacientes mayores de 80 años: tratamiento quirúrgico convencional en 80 casos consecutivos
}

\author{
Francisco Valdés $E^{1,3}$, Michel Bergoeing $R^{1,3}$, \\ Albrecht Krämer Sch ${ }^{1,3}$, Renato Mertens $M^{1,3}$, \\ Roberto Canessa $\mathbf{B}^{2}$, G uillermo Lema $\mathrm{F}^{2}$, \\ Bernardita $G$ arayar $\mathbf{P}^{1}$, Jorge U rzúa U ${ }^{2}$.
}

\section{Results of the surgical management of abdominal aortic aneurysms in 80 patients over 80 years of age}

Background: Abdominal aortic aneurysms (AAA) may be lethal unless appropriately and timely treated. Since age is a surgical risk, octogenarians are usually not considered as candidates for surgical intervention. Aim: To asses surgical complications and mortality in octogenarians treated for AAA. Subjects and Methods: Patients aged 80 years older, treated consecutively between 1984-2001 were retrospectively analyzed. Results: Sixty one patients were male, and their age ranged from 80 to 95 years. All were treated with open surgery. The operation was elective in 58 and as an emergency in 22 patients (symptomatic or ruptured AAA). Aortic diameter was $6.8 \pm 1.4 \mathrm{~cm}$ in asymptomatic patients and $7.7 \pm 1.8 \mathrm{~cm}$ in emergency cases $(\mathrm{p}=0.024)$. Thirty days postoperative mortality was $5.1 \%$ in elective surgery compared to $40.6 \%$ in emergency operations ( $p<0.01$ ). Five years survival rate was $44.7 \%$ in asymptomatic patients compared to $10.4 \%$ in the emergency cases ( $p<0.023$ ). Conclusions: Elective surgery for asymptomatic AAA can be performed with low operative mortality in octogenarians. However, surgery in emergency cases has an 8 fold increase in risk. Accordingly, octogenarian patients should be considered for elective AAA repair in a selective basis (Rev Méd Chile 2003; 131: 981-6).

(Key Words: Aged, 80 and over; Aneurysm, dissecting; Surgical procedures, operative)

Recibido el 18 de marzo, 2003. Aceptado en versión corregida el 17 de julio, 2003. Departamentos de Enfermedades Cardiovasculares ${ }^{1}$, Anestesia ${ }^{2}$ y División de Cirugía ${ }^{3}$, Facultad de Medicina y Hospital Clínico Pontificia Universidad Católica de Chile.

Correspondencia a: Dr. Francisco Valdés E. Marcoleta 367 $6^{\circ}$ piso. Teléfono: 3543382. Fax: 6326812. E mail: franval@med.puc.cl 
T a incidencia de aneurisma aórtico abdominal ـ(AAA) aumenta con la edad, alcanzando su máximo a los 80 años ${ }^{1}$. A la vez, datos poblacionales señalan que la esperanza de vida a los 80 años puede ser hasta 7 años ${ }^{2}$, por lo que el hallazgo de un AAA en estos pacientes plantea un dilema terapéutico. La mortalidad asociada a la cirugía por AAA ha disminuido drásticamente en las últimas décadas por el progreso continuo en el diagnóstico, manejo de las patologías asociadas, técnica operatoria y anestesia ${ }^{3-6}$. Sin embargo, la mortalidad persiste elevada en grupos específicos, particularmente en los pacientes de mayor edad ${ }^{3-10}$. El objetivo del presente trabajo es evaluar nuestros resultados con la cirugía convencional del AAA efectuada en forma electiva o de urgencia, en pacientes de 80 o más años de edad.

\section{Pacientes y MÉtodos}

Analizamos retrospectivamente los antecedentes clínicos de todos los pacientes de edad igual o mayor a 80 años, operados consecutivamente por AAA en nuestro Servicio entre enero de 1984 y agosto de 2001. Todos fueron operados en forma convencional. En dicho período no hubo pacientes octogenarios tratados por vía endovascular. Los datos fueron obtenidos de nuestra base de datos computacional, la que incluye las características demográficas, clínicas, tipo de tratamiento efectuado, complicaciones observadas y mortali- dad hospitalaria o a los 30 días. El seguimiento se efectuó por contacto personal con el paciente, sus familiares directos, su médico tratante 0 a través del Servicio de Registro Civil e Identificación para verificar data y causa de fallecimiento.

Los datos de sobrevida fueron incorporados a una tabla actuarial según el método de KaplanMeier. Como referente utilizamos las tablas de sobrevida actuarial para una población chilena pareada por sexo y edad ${ }^{2}$. El análisis estadístico se llevó a cabo con el programa computacional "StatView" ${ }^{\circledR}$ versión 5.0.1 (SAS Institute Inc. EE.UU). Para el análisis de diferencias significativas utilizamos las pruebas de chi-cuadrado, exacta de Fisher, Anova y log-rank. Consideramos significativa una $\mathrm{p}<0,05$.

\section{Resultados}

Durante los 17 años que abarca el presente estudio, fueron operados un total de 80 pacientes de 80 años o más (Figura 1). En el mismo período operamos un total de 664 pacientes, por lo que la presente experiencia constituye $12 \%$ de todos los pacientes operados por AAA. Cincuenta y ocho pacientes $(72,5 \%)$ se presentaron en forma asintomática y fueron intervenidos electivamente. Veintidós (27,5\%) fueron operados de urgencia: 9 por síntomas clínicos de expansión y 13 por franca ruptura. Las características demográficas y los antecedentes clínicos se detallan en la Tabla 1.

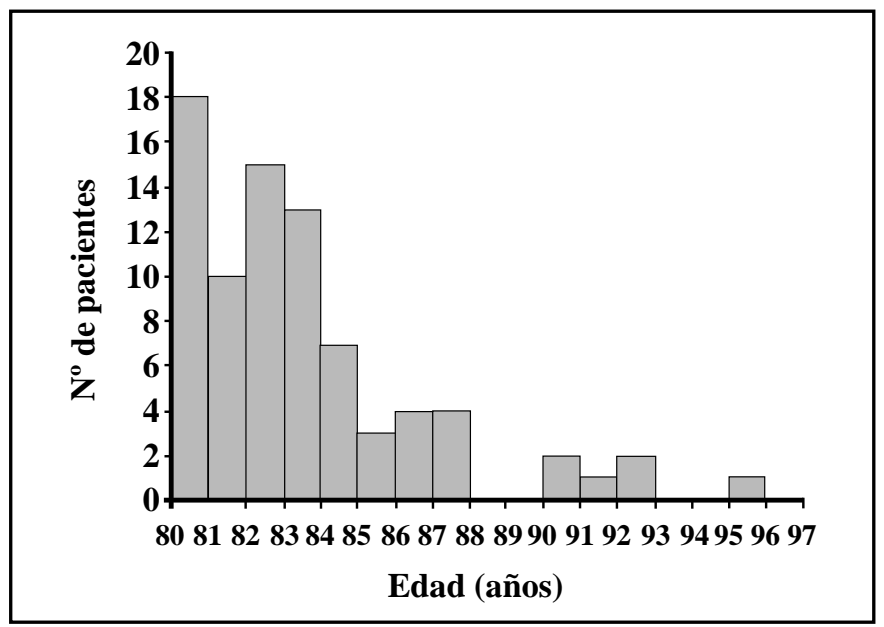

Figura 1. Distribución etaria de pacientes octogenarios portadores de AAA, operados entre 1984 y 2001. 
Tabla 1. C aracterísticas clínicas y demográficas de 80 pacientes octogenarios operados por AAA

\begin{tabular}{|lcc|}
\hline Variable & $\mathrm{n}$ & $\%$ \\
\hline Sexo & & \\
Masculino & 61 & 76,3 \\
Femenino & 19 & 23,7 \\
Presentación & 58 & 72,5 \\
$\quad$ Asintomático & 22 & 27,5 \\
Complicado (sintomático o roto) & & \\
Patologías asociadas & 22 & 27,5 \\
Aneurisma ilíaco & 19 & 23,7 \\
Cardiopatía coronaria† & 15 & 18,7 \\
Hipertensión arterial & 14 & 17,5 \\
Tabaquismo crónico & 7 & 8,7 \\
Arritmia & 7 & 8,7 \\
Enfermedad pulmonar obstructiva crónica & & \\
\hline
\end{tabular}

†2 pacientes revascularizados con anterioridad.

El tamaño del AAA difirió significativamente entre los casos asintomáticos y los complicados: $6,8 \pm 1,4$ $\mathrm{cm}$ vs $7,7 \pm 1,8 \mathrm{~cm} \quad(\mathrm{p}=0,024)$ (Figura 2$)$. Seis pacientes intervenidos electivamente y 2 con AAA roto se operaron por AAA de diámetro entre $5 \mathrm{y}$ $5,5 \mathrm{~cm}$.

El tipo de reconstrucción y procedimientos asociados se exponen en la Tabla 2. La mortalidad global de la serie (operaciones electivas y urgentes) fue $15 \%$ (12 pacientes). Para la cirugía electiva la mortalidad fue $5,1 \%$ ( 3 pacientes), mientras que para

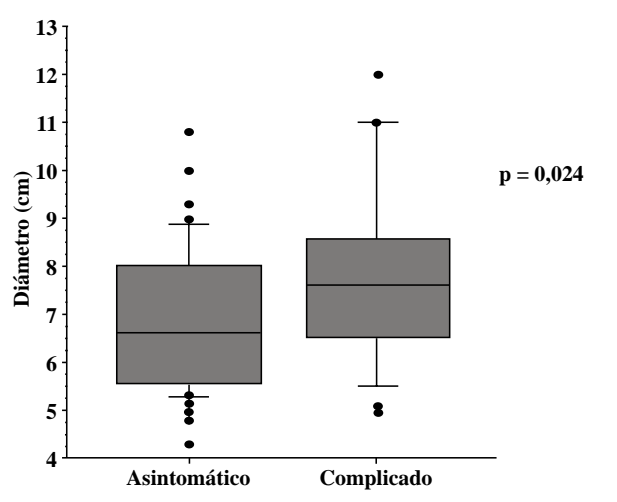

Figura 2. Diámetro del aneurisma según presentación clínica. la cirugía de urgencia la mortalidad fue 40,6\% (9 pacientes) ( $p=0,0003)$, correspondiendo a $33 \%$ de los AAA sintomáticos intactos y a $46 \%$ de los AAA complicados por ruptura. Las causas de mortalidad perioperatoria se muestran en la Tabla 3.

Seis pacientes se perdieron durante el seguimiento (4 en el grupo asintomático y 2 en el grupo complicado), lo que representa $7,7 \%$ de los sobrevivientes. El seguimiento promedio para los casos operados en forma electiva fue de $45,2 \pm 31,8$ meses $(0,7-147,3)$, y para los intervenidos de urgencia fue de $32,6 \pm 28,9(2,3-85,5)$.

$\mathrm{Al}$ comparar la sobrevida alejada de los pacientes asintomáticos versus los complicados, excluida la mortalidad perioperatoria, se observó que aquellos tienen una mayor sobrevida ( $41,2 \%$ vs $10,4 \%$ a 5 años, $p=0,0232$ ) (Figura 3). Si comparamos el grupo de pacientes asintomáticos con una población control pareada por sexo y edad, se observa una menor sobrevida a 5 años del grupo de estudio $(41,2 \%$ vs $63,8 \%)$, diferencia significativa $(p=0,0063)$ (Figura 4).

\section{DisCUSIÓN}

El progresivo aumento en la expectativa de vida de la población, la mayor incidencia de AAA con la edad y la mayor disponibilidad de medios de 
Tabla 2. Procedimientos realizados en 80 pacientes octogenarios operados por AAA

\begin{tabular}{|lrr|}
\hline Variable & $\mathrm{n}$ & $\%$ \\
\hline Tipo de reconstrucción & & \\
Puente aorto-aórticoł & 46 & 57,5 \\
Puente aorto-ilíaco* & 29 & 36,3 \\
Puente aorto-bifemoral & 3 & 3,8 \\
Puente axilo bifemoral y ligadura aórtica & 1 & 1,2 \\
Clampeo aórtico & 1 & 1,2 \\
Procedimientos asociados concomitantes & 5 & 6,2 \\
Reimplante arteria mesentérica inferior & 3 & 3,7 \\
Reimplante vasos renales & 3 & 3,7 \\
Exclusión hipogástrica & 2 & 2,5 \\
Endarterectomía femoral común & 1 & 1,2 \\
Reimplante arteria mesentérica superior & 1 & 1,2 \\
Nefrectomía izquierda incidental & 1 & 1,2 \\
Esplenectomía & 1 & 1,2 \\
Revascularización miocárdica & & \\
\hline
\end{tabular}

†11 pacientes abordaje retroperitoneal. ${ }^{\ddagger} 1$ paciente presentó AAA supra e inframenal. ${ }^{*} 1$ pacientes abordaje retroperitoneal.

Tabla 3. Causas de mortalidad operatoria en pacientes octogenarios operados por AAA

\begin{tabular}{|llc|}
\hline Variable & $\mathrm{n}$ & $\%$ \\
\hline Cirugía electiva (58 casos) & & \\
Sepsist* & 2 & 3,4 \\
Infarto agudo al miocardio & 1 & 1,7 \\
Cirugía de urgencia (22 casos) & 3 & \\
Paro intraoperatorio & 2 & 9,6 \\
Neumopatía & 1 & 4,5 \\
Infarto agudo al miocardio & 1 & 4,5 \\
Insuficiencia cardíaca & 1 & 4,5 \\
Shock séptico & 1 & 4,5 \\
Falla orgánica múltiple & 1 & \\
\hline
\end{tabular}

${ }^{\dagger} 1$ paciente por sepsis urinaria posterior al alta. ${ }^{*} 1$ paciente por colitis isquémica.

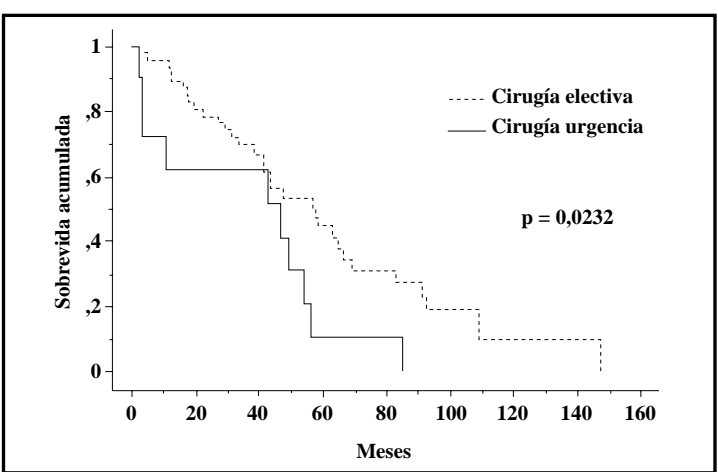

Figura 3. Sobrevida alejada de los pacientes operados en forma electiva versus los operados de urgencia, sin incluir la mortalidad operatoria.

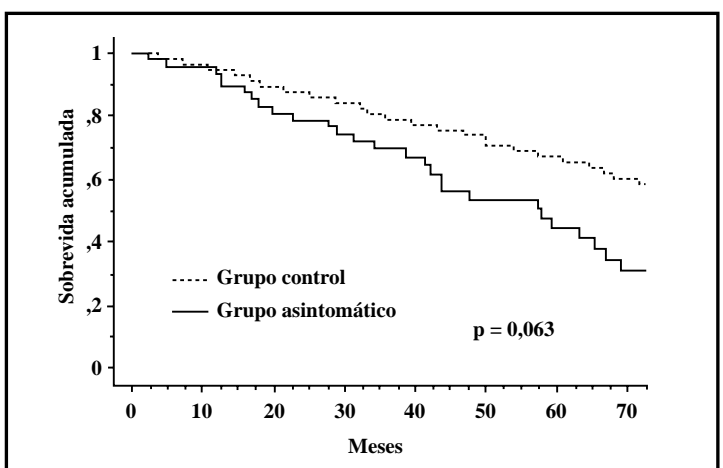

Figura 4. Sobrevida postoperatoria alejada del grupo de pacientes asintomáticos versus una población control pareada por sexo y edad, sin incluir mortalidad operatoria. 
diagnóstico por imágenes, ha incidido en un diagnóstico más frecuente de $\mathrm{AAA}^{11}$.

La reparación quirúrgica del AAA no está exenta de morbilidad y mortalidad, más aún si se realiza en condiciones de urgencia por ruptura. La cirugía electiva en poblaciones no seleccionadas, tiene una mortalidad que varía entre $0,72 \%$ y $5,0 \%$ en centros especializados ${ }^{5-7}$, mientras que en la cirugía de urgencia la mortalidad operatoria reportada alcanza entre $24 \%$ y $90 \% 4,10,12$, variando según si se trata de centros de referencia, hospitales generales o análisis poblacional. La edad es considerada habitualmente un factor de riesgo importante en todas las experiencias comunicadas, por lo que hay quienes estiman que la cirugía estaría contraindicada en octogenarios. Sin embargo, hay suficiente evidencia de que la morbimortalidad depende en parte de factores predecibles, modificables o controlables ${ }^{3-}$ 6,8 . Los resultados mostrados en la presente serie son comparables con los publicados en series similares, y están dentro de los estándares internacionales para la cirugía del AAA en poblaciones no seleccionadas $7,9,10$.

Estudios poblacionales de pacientes no operados del AAA confirman la relación entre diámetro y riesgo de ruptura, reportándose un riesgo a 3 años de $28 \%$ en lesiones de diámetro entre 5 y 6 $\mathrm{cm}$ y $41 \%$ en AAA de diámetro $>6 \mathrm{~cm}^{13}$. En un estudio prospectivo de 106 pacientes con AAA mayor a $5,5 \mathrm{~cm}$ que rechazaron la cirugía, la sobrevida a 3 años fue 17\%, falleciendo el 50\% por ruptura a los 9 meses (mediana) ${ }^{14}$.

Publicaciones recientes demuestran que AAA pequeños pueden ser observados periódicamente, con controles clínicos estrictos, sin una tasa mayor de ruptura si se intervienen aquellos que presentan crecimiento acelerado o alcanzan un diámetro igual o superior a 5,5 $\mathrm{cm}^{15,16}$. En la presente serie, el tamaño promedio del AAA en pacientes operados electivamente fue $6,8 \pm 1,4 \mathrm{~cm}$, y en los complicados $7,7 \pm 1,8 \mathrm{~cm}$, lo que revela una actitud conservadora en la indicación operatoria en este segmento etario. Cabe destacar que 2 pacientes en el grupo complicado fueron intervenidos por nuptura de un AAA menor a 5,5 cm.

Al comparar la sobrevida de los pacientes intervenidos en forma electiva con aquella en una población control, se observa que su expectativa de vida a 5 años es menor, como cabe esperar en un grupo de pacientes afectados de diversas patologías asociadas (hipertensión arterial, arrit- mias, aterosclerosis oclusiva, bronquitis obstructiva, insuficiencia renal, entre otras).

La mayor mortalidad operatoria, así como la menor esperanza de vida observada en pacientes octogenarios operados de urgencia por complicación del AAA, debiera promover el estudio y la evaluación para un tratamiento electivo del AAA en este grupo de pacientes. El marcado deterioro de la esperanza de vida de los sobrevivientes a la complicación del AAA puede ser el resultado de una enfermedad inicialmente más compleja, con compromiso de múltiples sistemas, como también el resultado de complicaciones perioperatorias con deterioro de la reserva fisiológica del paciente, evitables con una reparación electiva.

Desde 1991 se ha desarrollado el tratamiento endovascular del AAA, técnica mínimamente invasiva, que ofrece una alternativa terapéutica que se basa en la exclusión del AAA aunque no lo elimina, y que tiene una menor morbilidad ${ }^{17}$. Dicha técnica, en uso en nuestro medio desde $1997^{18,19}$, en un estudio comparativo retrospectivo con la cirugía convencional en pacientes octogenarios, no mostró diferencia en la mortalidad operatoria, sin embargo tuvo un impacto significativo en la morbilidad perioperatoria y, por ende, en la recuperación de la calidad de vida de los pacientes ${ }^{20}$. Esta técnica resulta especialmente atractiva en pacientes de alto riesgo con anatomía favorable. La mayor difusión del tratamiento endovascular depende de una capacitación adecuada de los especialistas para efectuarlo, del conocimiento más preciso de las complicaciones y la capacidad para resolverlas, de los resultados alejados y, no menos importante, de la progresiva reducción de sus costos.

\section{CONCLUSIONES}

Concluimos que los pacientes octogenarios deben ser evaluados en forma crítica para enfrentar en forma electiva el tratamiento de su AAA, en especial si éste supera los 5,5 cm de diámetro. La cirugía convencional electiva tiene, en nuestra experiencia, una baja mortalidad de acuerdo a los estándares intemacionales para la cirugía de esta grave lesión. En la medida que se reduzcan los costos y se perfeccionen los dispositivos para la cinugía endovascular, este grupo creciente de pacientes tendrá mejores alternativas de tratamiento. 


\section{REFERENCIAS}

1. Bengtsson $H$, Bergqvist $D$, Sternby $N$. Increasing prevalence of abdominal aortic aneurysms. A necropsy study. Eur J Surg 1992; 158: 19-23.

2. Centro Latinoamericano y Caribeño de Demografía - CELADE. Boletín demográfico $\mathrm{N}^{\circ}$ 67. América Latina: Tablas de Mortalidad, 19502025. CELADE; enero 2001.

3. Crawford E, Saleh S, Babb J, Glaeser D, Vaccaro P, Silvers A. Infrarenal abdominal aortic aneurysm. Factors influencing survival after operation performed over a 25 years period. Ann Surg 1981; 193: 699-708.

4. Katz D, Stanley J, Zelenock G. Operative mortality rates for intact and ruptured abdominal aortic aneurysms in Michigan: an eleven years statewide experience. J Vasc Surg 1994; 19: 804-17.

5. Vaidés F, Krämer A, Mertens R, Santini A, Canessa R, LEMA G ET AL. Aneurisma aórtico abdominal: evolución de la morbimortalidad de la cirugía electiva durante 20 años. Rev Méd Chile 1997; 125: 425-32.

6. Hertzer N, Mascha E, Karafa M, O’Hara P, Krajewski L, Beven E. Open infrarenal abdominal aortic aneurysm repair: The Cleveland Clinic experience from 1989 to 1998. J Vasc Surg 2002; 35: 1145-54.

7. O'Hara P, Hertzer N, Krajewski L, Tan M, Xiong X, Beven E. Ten years experience with abdominal aortic aneurysm repair in octogenarians: early results and late outcome. J Vasc Surg 1995; 21: 830-8.

8. Dardik A, Lin J, Gordon T, Wimams M, Perler B. Results of elective abdominal aortic aneurysm repair in the 1990's: a population based analysis of 2335 cases. J Vasc Surg 1999; 30: 985-95.

9. Dean R, Woody J, Enarson C, Hansen K, Plonk G. Operative treatment of abdominal aortic aneurysms in octogenarians. When is it too much too late? Ann Surg 1993; 217: 721-8.

10. Kazmers A, Perkins A, Jacobs L. Outcomes after abdominal aortic aneurysm repair in those $\geq 80$ years of age: recent veterans affairs experience. Ann Vasc Surg 1998; 12: 106-12.

11. Nevitt M, Ballard D, Halett J. Prognosis of abdominal aortic aneurysms. A population based study. N Engl J Med 1989; 321: 1009-14.

12. Ingoldby C, Wujanto R, Mitchell J. Impact of vascular surgery on community from ruptured aortic aneurysms. Br J Surg 1986; 73: 551-3.

13. Jones A, Cahill D, Gardham R. Outcome in patients with a large abdominal aortic aneurysm considered unfit for surgery. Br J Surg 1998; 85: 1382-4.

14. Conway K, Byrne J, Townsend M, Lane I. Prognosis of patients turned down for conventional abdominal aortic aneurysm repair in the endovascular and sonographic era: Szilagyi revisited?J Vasc Surg 2001; 33: 752-77.

15. Lederle F, Wilson S, Johnson G, Reinke D, LtTody F, ACHER C ET AL. Immediate repair compared with surveillance of small abdominal aortic aneurysms. N Engl J Med 2002; 346: 1437-44.

16. The United Kingdom Small Aneurysm Trial Participants. Long term outcomes of immediate repair compared with surveillance of small abdominal aortic aneurysms. N Engl J Med 2002; 346: 1445-52.

17. Parodi J, Palmaz J, Barone H. Transfemoral intraluminal graft implantation for abdominal aortic aneurysm. Ann Vas Surg 1991; 5: 491-9.

18. Valdés F, Seitz J, Fava M, Krämer A, Mertens R, Espíndola M ET aL. Tratamiento del aneurisma aórtico abdominal por vía endovascular: Experiencia inicial. Rev Méd Chile 1998; 126: 1206-15.

19. Valdés F, Seitz J, Mertens R, Espíndola M, Krämer A, FAVA M ET AL. Tratamiento endovascular de aneurisma aórtico abdominal: resultados a 24 meses. Rev Chil Cardiol 1999; 18: 153-4.

20. Sicard G, Rubin B, Sánchez L, Keluer C, Wayne MF, Picus D ET AL. Endoluminal graft repair for abdominal aortic aneurysms in high risk patients and octogenarians. Ann Surg 2001; 234: 427-37. 